J Am Chem Soc. 2019 July 17; 141(28): 10958-10961. doi:10.1021/jacs.9b04424.

\title{
SLIRP Interacts with Helicases to Facilitate 2' -0 -Methylation of rRNA and to Promote Translation
}

\author{
Lin Li, Weili Miao, Preston Williams, Cheng Guo, Yinsheng Wang ${ }^{*}$ \\ Department of Chemistry, University of California Riverside, Riverside, California 92521-0403, \\ United States
}

\begin{abstract}
SRA stem-loop-interacting RNA-binding protein (SLIRP) is a versatile protein that can interact with the stem-loop structure in RNA and with G quadruplex DNA. By using a quantitative proteomic experiment, we found that SLIRP interacts with the majority of the human helicase proteome. We also found that these interactions facilitate $2^{\prime}-O$-methylation of a number of nucleosides in rRNA and promote protein translation. Hence, we uncovered a novel function of SLIRP protein and offered novel mechanistic insights into its function as a RNA chaperone and into the regulation of $2^{\prime}-O$-methylation of rRNA.
\end{abstract}

The SRA stem-loop-interacting RNA-binding protein (SLIRP) was initially discovered as a protein that can interact with a functional substructure of SRA (STR7) and repress nuclear receptor transactivation. ${ }^{1}$ Endogenous SLIRP is localized mainly in the mitochondria, ${ }^{1}$ where it plays an indispensable role in maintaining mitochondria-localized mRNA transcripts encoding for proteins involved in oxidative phosphorylation. ${ }^{2}$ In addition, our recent quantitative proteomic experiment revealed that SLIRP can bind directly with G quadruplex (G4) DNA in vitro and binds with guanine-rich regions in chromatin that can potentially fold into $\mathrm{G} 4$ structures. $^{3}$

SLIRP forms a complex with leucine-rich pentatricopeptide repeat-containing protein (LRPPRC) and protects the latter from degradation. ${ }^{4,5}$ The SLIRP-LRPPRC complex promotes the polyadenylation and coordination of translation of mitochondrial mRNA. ${ }^{6,7}$ The protein complex was recently found to bind throughout the mitochondrial transcriptome, especially the mRNAs, and act as a global RNA chaperone that stabilizes RNA structures to allow for exposure of the regions of RNA that are necessary for stabilization, polyadenylation, and translation. ${ }^{8}$

Herein, we set out to explore novel functions of SLIRP by characterizing systematically the interaction proteome of SILRP. We utilized a quantitative proteomic method, relying on stable isotope labeling by amino acid in cell culture (SILAC), to characterize, at the

\footnotetext{
*Corresponding Author: Yinsheng.Wang@ucr.edu.

Supporting Information

The Supporting Information is available free of charge on the ACS Publications website at DOI: 10.1021/jacs.9b04424.

Detailed experimental procedures and supplementary data (PDF)

The authors declare no competing financial interest.
} 
proteome-wide level, the SLIRP-binding proteins. Toward this goal, we used our previously reported CRISPR-engineered HEK293T cells which carry $3 \times$ FLAG and $2 \times$ Strept tags fused to the C-terminus of endogenous SLIRP protein (SLIRP-TAPTAG cells). ${ }^{3}$ To remove experimental bias, we performed SILAC experiments with both forward and reverse labelings (Figure 1).

The liquid chromatography-tandem mass spectrometry (LCMS/MS) results revealed 279 proteins showing binding preference toward SLIRP, with SILAC ratios being $\geq 2$ (Table S1 and Figure 1b). Gene ontology (GO) function analysis revealed the enrichment of helicases and those proteins that function in RNA binding and rRNA binding (Figure 1c and Figure S1). In addition, our proteomic data confirmed a previously reported interaction partner of SLIRP, i.e., LRPPRC, with an average SILAC ratio of 46.7 (Figure S2 and Table S1).

To explore further the degree to which the SLIRP interacts with helicase proteins, we employed a previously reported SILAC coupled with LC-PRM (PRM: parallel reaction monitoring) method, ${ }^{9}$ which allows for the quantitative assessment of 121 distinct human helicases, to monitor systematically the binding between SLIRP and helicase proteins. Our results revealed that, among the 121 distinct human helicases monitored, 78 could be detected in the SLIRP pull-down experiment. Strikingly, 70 out of the 78 helicases were enriched by at least 2-fold with the SLIRP pull-down relative to the control (Figure 2, Figure S3 and Table S2). In this vein, our quantitative proteomic data unveiled $\geq 10$-fold enrichments of DDX17 and DDX21 in the SLIRP pull-down sample relative to the control pull-down (Figure 3a,b).

DDX17, DDX21 and NOP58 were previously shown to function in rRNA processing. ${ }^{10-14}$ We also validated the interactions between SLIRP and DDX17/DDX21/NOP58 by pulldown followed by Western blot analyses (Figure 3c,d). In addition, treatment with RNase A led to diminished interactions between SLIRP and the three proteins, indicating that the SLIRP-DDX17/DDX21/NOP58 interactions are RNA-dependent (Figure 3c). The reciprocal experiment using Flag-tagged DDX17/DDX21 further confirmed their interactions with SLIRP in cells (Figure 3d).

DDX21 was found to participate in $2^{\prime}-O$-methylation of rRNA. ${ }^{11,12}$ Along this line, we found that Box C/D RNP proteins (e.g., NOP58, NOP56 and FBL), which are involved with $2^{\prime}$ - $O$-methylation of rRNA, ${ }^{13,14}$ are also enriched in the pull-down experiment (Figure S1 and Table S1). Hence, we next asked whether SLIRP also modulates the $2^{\prime}-O$-methylation of rRNA. To this end, we monitored site-specific $2^{\prime}-O$-methylation in rRNA by real-time quantitative polymer-ase chain reaction (RT-qPCR), which is on the basis of the principle that $2^{\prime}-O$-methylation inhibits reverse transcription in the presence of low concentrations of dNTPs. ${ }^{11,15}$ The results showed that the levels of $2^{\prime}$ - $O$-methylation at C3848, G4464, U4197 in 28S rRNA and U1805 in 18S rRNA were significantly diminished upon shRNAmediated knockdown of SLIRP, or upon knockdown of NOP58 or DDX21 (Figure 4). Nevertheless, the levels of $2^{\prime}-O$-methylation at $\mathrm{C} 1703$ in $18 \mathrm{~S}$ rRNA and $\mathrm{C} 4506$ in $28 \mathrm{~S}$ rRNA were not altered upon knockdown of SLIRP (Figure 4). In this vein, it is of note that knockdown of SLIRP or NOP58 did not affect each other's expression (Figure S4). 
We also conducted RiboMethSeq analysis to assess the alterations in site-specific $2^{\prime}-O$ methylation in rRNA after SLIRP knockdown. ${ }^{16,17}$ We found that the changes in $2^{\prime}$ - $O$ methylation obtained from RiboMethSeq were consistent with the aforementioned RT-qPCR results (Figure $4 \mathrm{~b}$ and Figure S5). In addition, we measured, by using LC-MS/MS, the global levels of $2^{\prime}-O$-methylated nucleosides and pseudouridine in rRNA after knockdown of SLIRP (Figure S6). It turned out that there were no significant alterations in the levels of $2^{\prime}$-O-methylated nucleosides or pseudouridine after knockdown of SLIRP (Figure S6). This is in agreement with the results obtained from RiboMethSeq analysis (Figure 4a and Figure S5), indicating that the SLIRP-mediated $2^{\prime}$ - $O$-methylation is site-specific and is perhaps modulated by local secondary structure of rRNA (Figure S7). ${ }^{18}$ In this context, we also examined the locations of the sites with altered levels of $2^{\prime}-O$-methylation in the ribosome structure, ${ }^{18}$ and it turned out that the affected sites were localized throughout the ribosome structure, where some are localized close to critical regions such as the A-sites (28S A3697, 28S U4197) and intersubunit bridge regions (18S U1804, 28S G2863) (Figure S7).

$2^{\prime}$-O-Methylation of rRNA contributes to the assembly of functional ribosomes and regulates the efficiency of translation. ${ }^{14,19}$ We reason that the SLIRP-mediated $2^{\prime}$ - $O$ methylation of rRNA may also modulate the rate of translation. To test this, we monitored, by using a luciferase reporter assay, the translation rate after knockdown of SLIRP, NOP58 or DDX21 (Figure 5). Our results revealed a significantly lower translation rate in HEK293T cells upon shRNA-mediated knockdown of SLIRP, NOP58 or DDX21 relative to cells treated with control shRNA, while the mRNA transcription of the reporter gene was not altered (Figure $5 \mathrm{~b}$ and Figure S8), supporting that the SLIRP-mediated $2^{\prime}-O$-methylation of rRNA assumes important roles in translation regulation. In this regard, it is of note that the luciferase reporter assay only reveals cap-dependent translation, and it will be important to examine, in the future, if cap-independent translation is also influenced by knockdown of SLIRP.

Together, we characterized comprehensively, for the first time, the interaction proteome of SLIRP, and our proteomic data revealed a pronounced enrichment of helicase proteins as interaction partners of SLIRP. Our results provide plausible mechanistic insights into the recent observation that SLIRP, in conjunction with LRPPRC, serves as a RNA chaperone that modulates the secondary structure and stability of the entire mitochondrial transcriptome. ${ }^{8}$ In this respect, SLIRP may recruit RNA helicases to unwind and expose certain regions of mitochondrial RNA that are required for stabilization, polyadenylation and translation. Additionally, we uncovered a novel function of SLIRP in modulating translation by stimulating the $2^{\prime}-O$-methylation at several sites in rRNA through its binding with NOP58 and DDX21. SLIRP harbors a RNA recognition motif, which recognizes secondary structures in RNA. ${ }^{1}$ We speculate that SLIRP may also bind to structured regions of rRNA and recruit RNA helicase(s) to unwind these local structures and to facilitate $2^{\prime}-O$ methylation in rRNA. This may explain why SLIRP only modulates the $2^{\prime}-O$-methylation of selected sites in rRNA. Therefore, our work also revealed a new molecular determinant for $2^{\prime}$-O-methylation of rRNA.

Considering the recently discovered function of SLIRP in binding to G4 DNA, ${ }^{3}$ it can be envisaged that SLIRP may also help recruit helicase proteins to G4 DNA, thereby unwinding 
G4 DNA structures for supporting normal DNA metabolism (e.g., DNA replication, transcription and repair).

\section{Supplementary Material}

Refer to Web version on PubMed Central for supplementary material.

\section{ACKNOWLEDGMENTS}

This work was supported by the National Institutes of Health (R01ES025121).

\section{REFERENCES}

(1). Hatchell EC; Colley SM; Beveridge DJ; Epis MR; Stuart LM; Giles KM; Redfern AD; Miles LE; Barker A; MacDonald LM; Arthur PG; Lui JC; Golding JL; McCulloch RK; Metcalf CB; Wilce JA; Wilce MC; Lanz RB; O’Malley BW; Leedman PJ SLIRP, a small SRA binding protein, is a nuclear receptor corepressor. Mol. Cell 2006, 22, 657-68. [PubMed: 16762838]

(2). Baughman JM; Nilsson R; Gohil VM; Arlow DH; Gauhar Z; Mootha VK A computational screen for regulators of oxidative phosphorylation implicates SLIRP in mitochondrial RNA homeostasis. PLoS Genet. 2009, 5, No. e1000590. [PubMed: 19680543]

(3). Williams P; Li L; Dong X; Wang Y Identification of SLIRP as a G quadruplex-binding protein. J. Am. Chem. Soc 2017, 139, 12426-12429. [PubMed: 28859475]

(4). Sasarman F; Brunel-Guitton C; Antonicka H; Wai T; Shoubridge EA LRPPRC and SLIRP interact in a ribonucleoprotein complex that regulates posttranscriptional gene expression in mitochondria. Mol. Biol. Cell 2010, 21, 1315-23. [PubMed: 20200222]

(5). Lagouge M; Mourier A; Lee HJ; Spahr H; Wai T; Kukat C; Silva Ramos E; Motori E; Busch JD; Siira S; Kremmer E; Filipovska A; Larsson NG SLIRP Regulates the Rate of Mitochondrial Protein Synthesis and Protects LRPPRC from Degradation. PLoS Genet. 2015, 11, No. e1005423. [PubMed: 26247782]

(6). Chujo T; Ohira T; Sakaguchi Y; Goshima N; Nomura N; Nagao A; Suzuki T LRPPRC/SLIRP suppresses PNPase-mediated mRNA decay and promotes polyadenylation in human mitochondria. Nucleic Acids Res 2012, 40, 8033-47. [PubMed: 22661577]

(7). Ruzzenente B; Metodiev MD; Wredenberg A; Bratic A; Park CB; Camara Y; Milenkovic D; Zickermann V; Wibom R; Hultenby K; Erdjument-Bromage H; Tempst P; Brandt U; Stewart JB; Gustafsson CM; Larsson NG LRPPRC is necessary for polyadenylation and coordination of translation of mitochondrial mRNAs. EMBO J 2012, 31, 443-56. [PubMed: 22045337]

(8). Siira SJ; Spahr H; Shearwood AJ; Ruzzenente B; Larsson NG; Rackham O; Filipovska A LRPPRC-mediated folding of the mitochondrial transcriptome. Nat. Commun 2017, 8, 1532. [PubMed: 29146908]

(9). Miao W; Li L; Wang Y Identification of helicase proteins as clients for HSP90. Anal. Chem 2018, 90, 11751-11755. [PubMed: 30247883]

(10). Jalal C; Uhlmann-Schiffler H; Stahl H Redundant role of DEAD box proteins p68 (Ddx5) and p72/p82 (Ddx17) in ribosome biogenesis and cell proliferation. Nucleic Acids Res. 2007, 35, 3590-601. [PubMed: 17485482]

(11). Zhou F; Liu Y; Rohde C; Pauli C; Gerloff D; Kohn M; Misiak D; Baumer N; Cui C; Gollner S; Oellerich T; Serve H; Garcia-Cuellar MP; Slany R; Maciejewski JP; Przychodzen B; Seliger B; Klein HU; Bartenhagen C; Berdel WE; Dugas M; Taketo MM; Farouq D; Schwartz S; Regev A; Hebert J; Sauvageau G; Pabst C; Huttelmaier S; Muller-Tidow C AML1-ETO requires enhanced C/D box snoRNA/RNP formation to induce self-renewal and leukaemia. Nat. Cell Biol 2017, 19, 844-855. [PubMed: 28650479]

(12). Calo E; Flynn RA; Martin L; Spitale RC; Chang HY; Wysocka J RNA helicase DDX21 coordinates transcription and ribosomal RNA processing. Nature 2015, 518, 249-53. [PubMed: 25470060] 
(13). Newman DR; Kuhn JF; Shanab GM; Maxwell ES Box C/D snoRNA-associated proteins: two pairs of evolutionarily ancient proteins and possible links to replication and transcription. RNA 2000, 6, 861-79. [PubMed: 10864044]

(14). Erales J; Marchand V; Panthu B; Gillot S; Belin S; Ghayad SE; Garcia M; Laforets F; Marcel V; Baudin-Baillieu A; Bertin P; Coute Y; Adrait A; Meyer M; Therizols G; Yusupov M; Namy O; Ohlmann T; Motorin Y; Catez F; Diaz JJ Evidence for rRNA 2'-O-methylation plasticity: Control of intrinsic translational capabilities of human ribosomes. Proc. Natl. Acad. Sci. U.S. A 2017, 114, 12934-12939. [PubMed: 29158377]

(15). Marcel V; Ghayad SE; Belin S; Therizols G; Morel AP; Solano-Gonzalez E; Vendrell JA; Hacot S; Mertani HC; Albaret MA; Bourdon JC; Jordan L; Thompson A; Tafer Y; Cong R; Bouvet P; Saurin JC; Catez F; Prats AC; Puisieux A; Diaz JJ p53 acts as a safeguard of translational control by regulating fibrillarin and rRNA methylation in cancer. Cancer Cell 2013, 24, 318-30. [PubMed: 24029231]

(16). Marchand V; Ayadi L; El Hajj A; Blanloeil-Oillo F; Helm M; Motorin Y High-throughput mapping of 2'-O-Me residues in RNA using next-generation sequencing (Illumina RiboMethSeq Protocol). Methods Mol. Biol 2017, 1562, 171-187. [PubMed: 28349461]

(17). Birkedal U; Christensen-Dalsgaard M; Krogh N; Sabarinathan R; Gorodkin J; Nielsen H Profiling of ribose methylations in RNA by high-throughput sequencing. Angew. Chem., Int. Ed 2014, 54, 451-455.

(18). Khatter H; Myasnikov AG; Natchiar SK; Klaholz BP Structure of the human 80S ribosome. Nature 2015, 520, 640-5. [PubMed: 25901680]

(19). Baxter-Roshek JL; Petrov AN; Dinman JD Optimization of ribosome structure and function by rRNA base modification. PLoS One 2007, 2, No. e174. [PubMed: 17245450] 
a

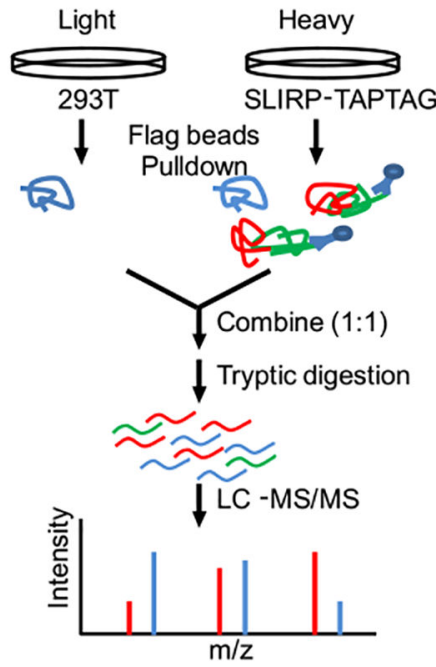

b

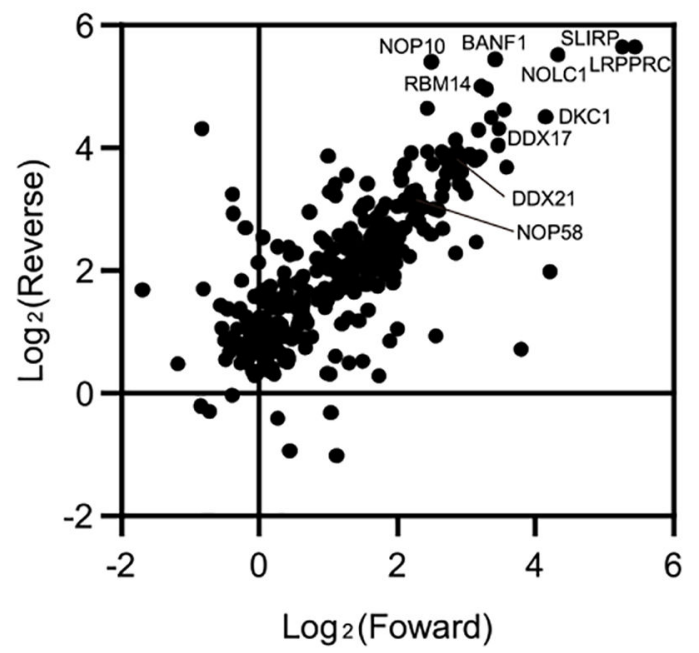

C

GO analysis

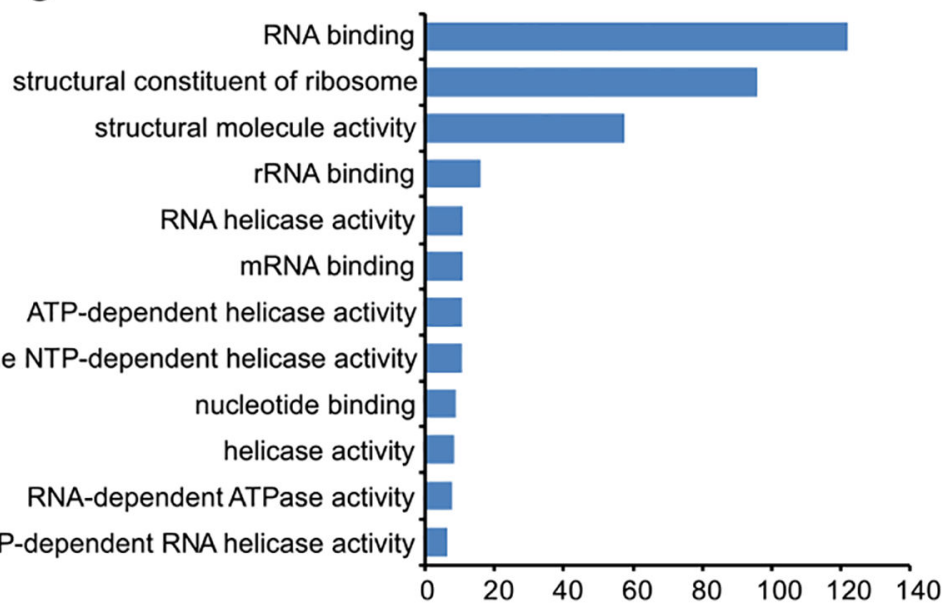

Figure 1.

Analysis of SLIRP-binding proteome. (a) A scheme depicting the procedures for the SILAC-based quantitative profiling of SLIRP-binding proteins. (b) A scatter plot showing the ratios obtained from forward and reverse SILAC labeling using shotgun proteomic analysis. (c) GO analysis of SLIRP-binding proteins. 

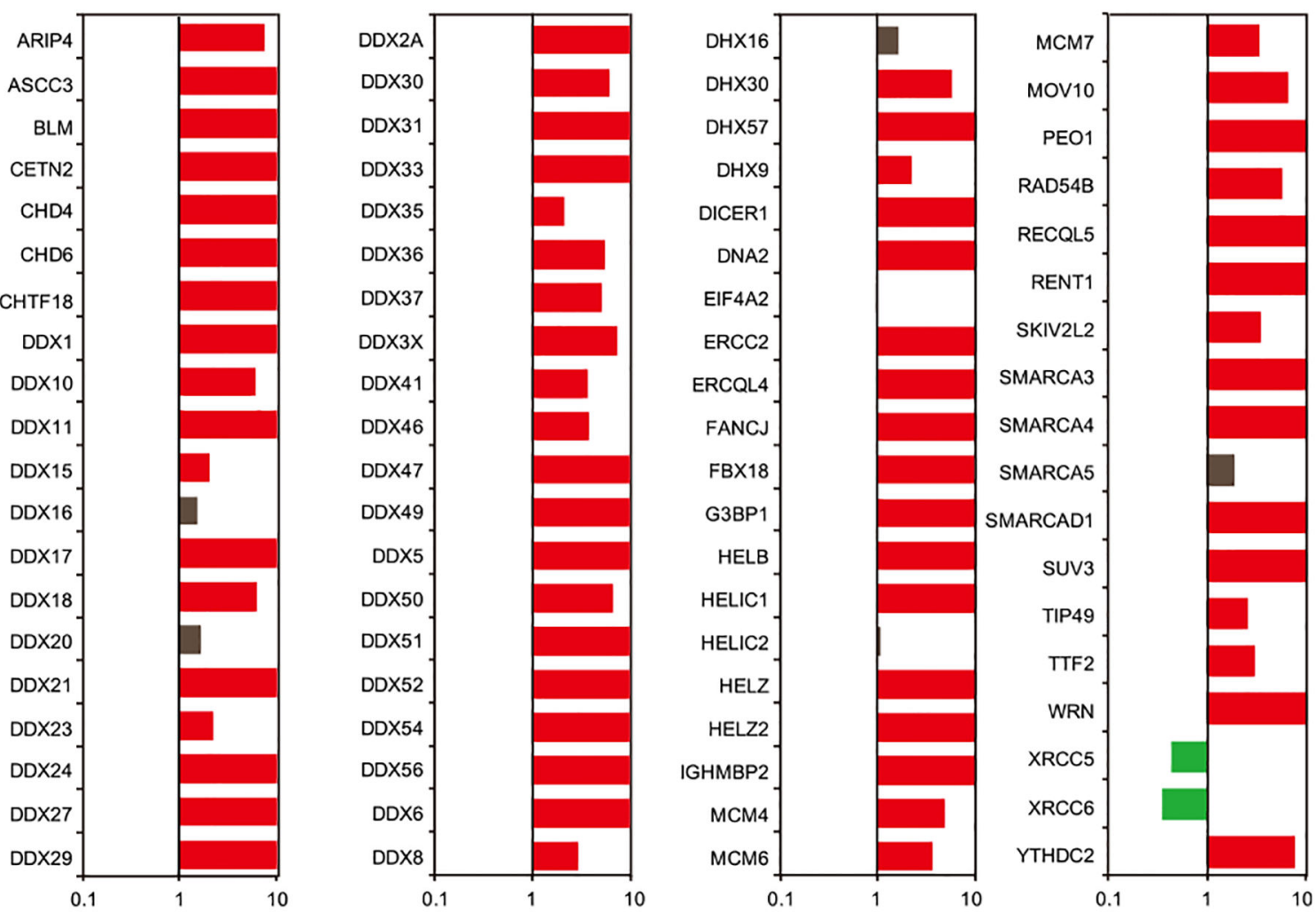

Figure 2.

PRM-based targeted proteomic analysis of the helicase proteins that interact with SLIRP.

Plotted are the ratios of levels of helicase proteins in SLIRP over control pull-down experiments. 

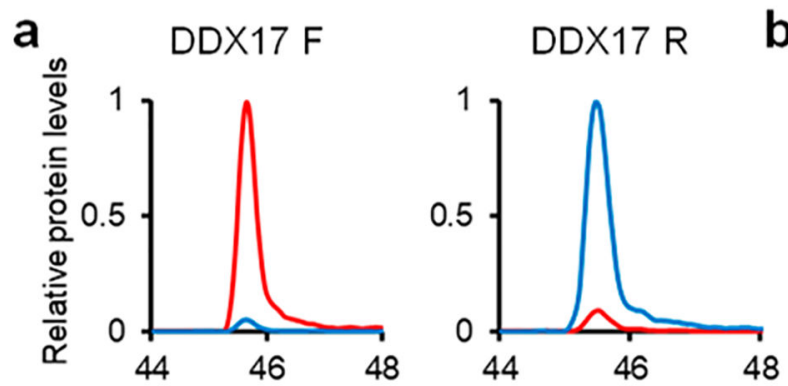

b

DDX21 F

DDX21 R

C
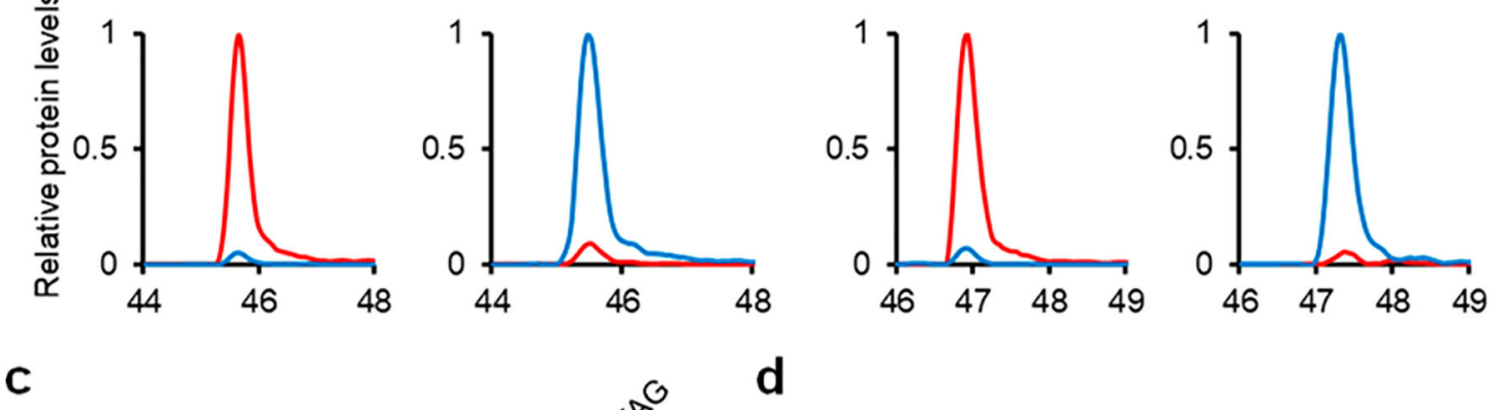

$\underline{\underline{a}}$

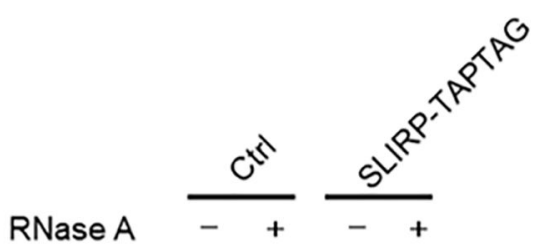

$+-+$
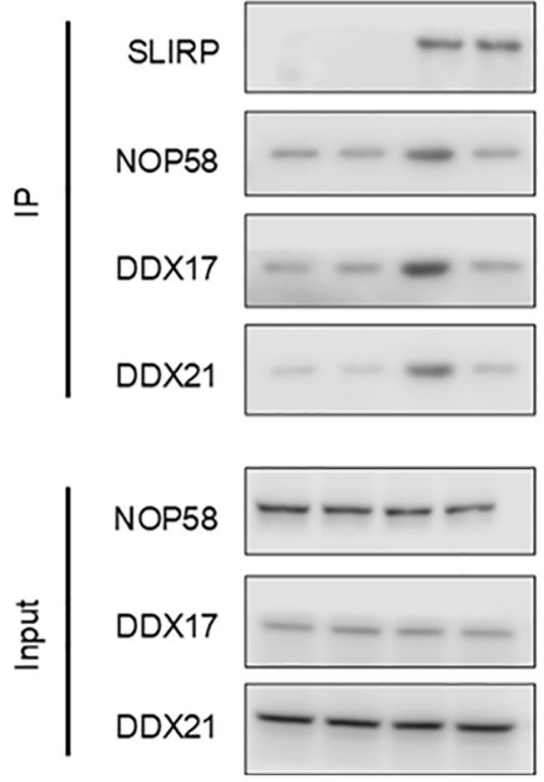

d
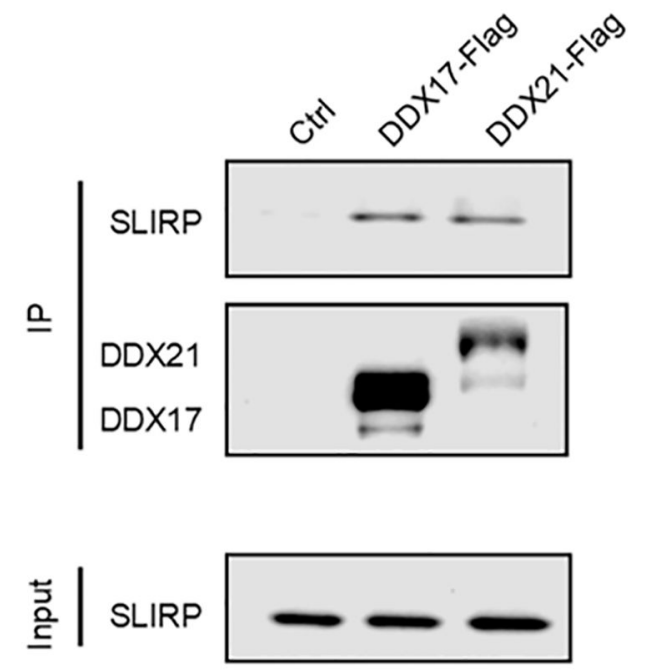

Figure 3.

Interactions between SLIRP and DDX17/DDX21/NOP58. (a,b) Representative PRM traces showing the relative enrichment of DDX17 (a) or DDX21 (b) from anti-Flag pull-down experiments using lysates of SLIRP-TAPTAG and parental HEK293T cells. " $F$ ' and 'R" designate forward and reverse SILAC labeling experiments, respectively. The red and blue traces in (a) and (b) from forward SILAC experiment represent pull-down samples from SLIRP-TAPTAG and parental HEK293T cells, respectively. The opposite color scheme was used for the reverse SILAC experiment. (c) Western blot results showing Flag pull-down using lysates of SLIRP-TAPTAG cells and HEK293T cells with or without RNase A treatment. (d) Western blot results showing Flag pull-down in HEK293T cells transfected with or without DDX17-Flag or DDX21-Flag. 
a
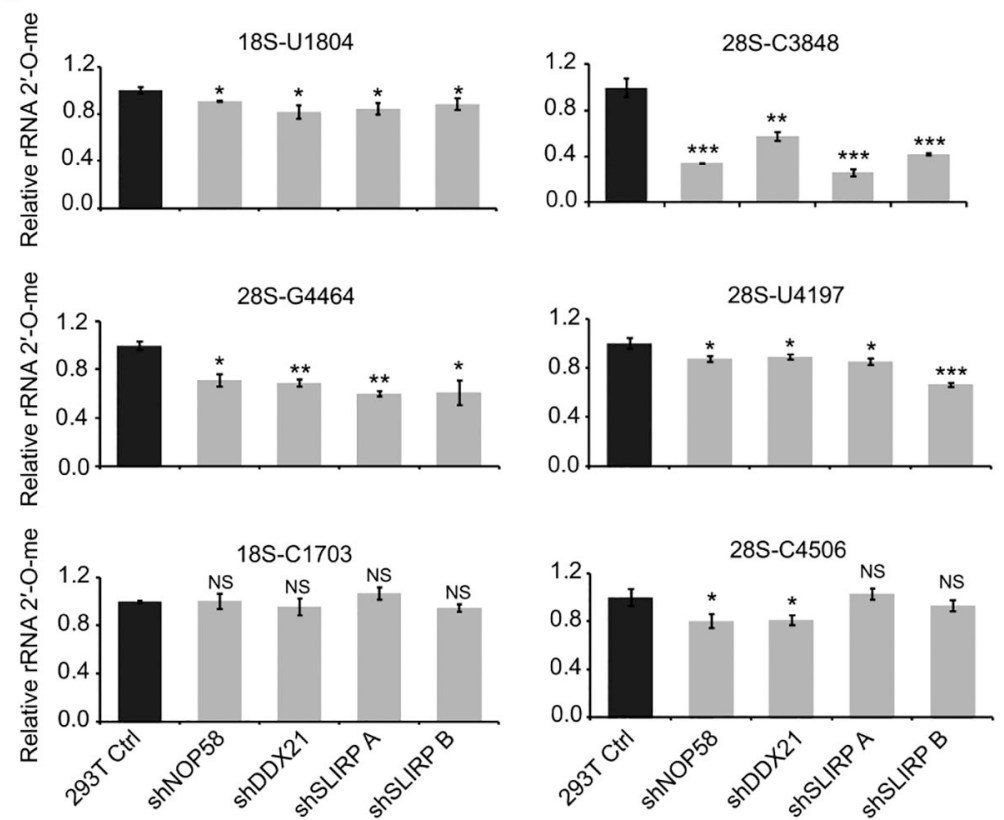

b

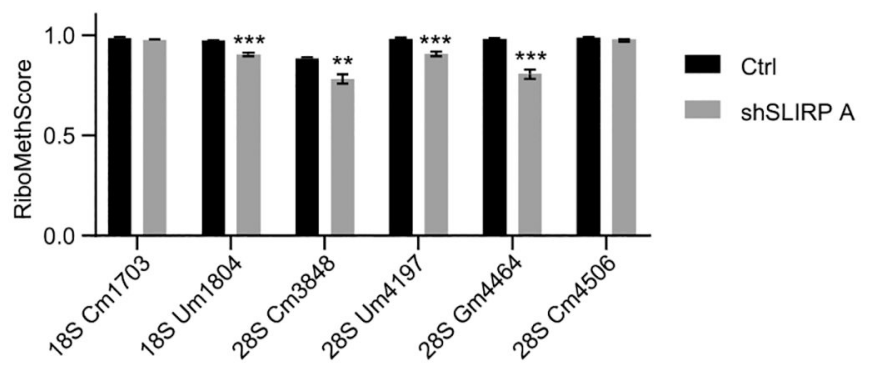

Figure 4.

SLIRP modulates $2^{\prime}-O$-methylation in rRNA. (a) Shown are the relative $2^{\prime}-O$-methylation levels at 6 sites on rRNA after shRNA-mediated knockdown of NOP58, DDX21, or SLIRP. (b) The mean RiboMethScore of individual sites on 18S and 28S rRNA obtained from RiboMethSeq analysis. Unpaired, two-tailed Student's t-test was employed to determine the p values: *, $p<0.05 ; * *, p<0.01 ; * * *, p<0.001$; NS, $p>0.05(n=3)$. 
a
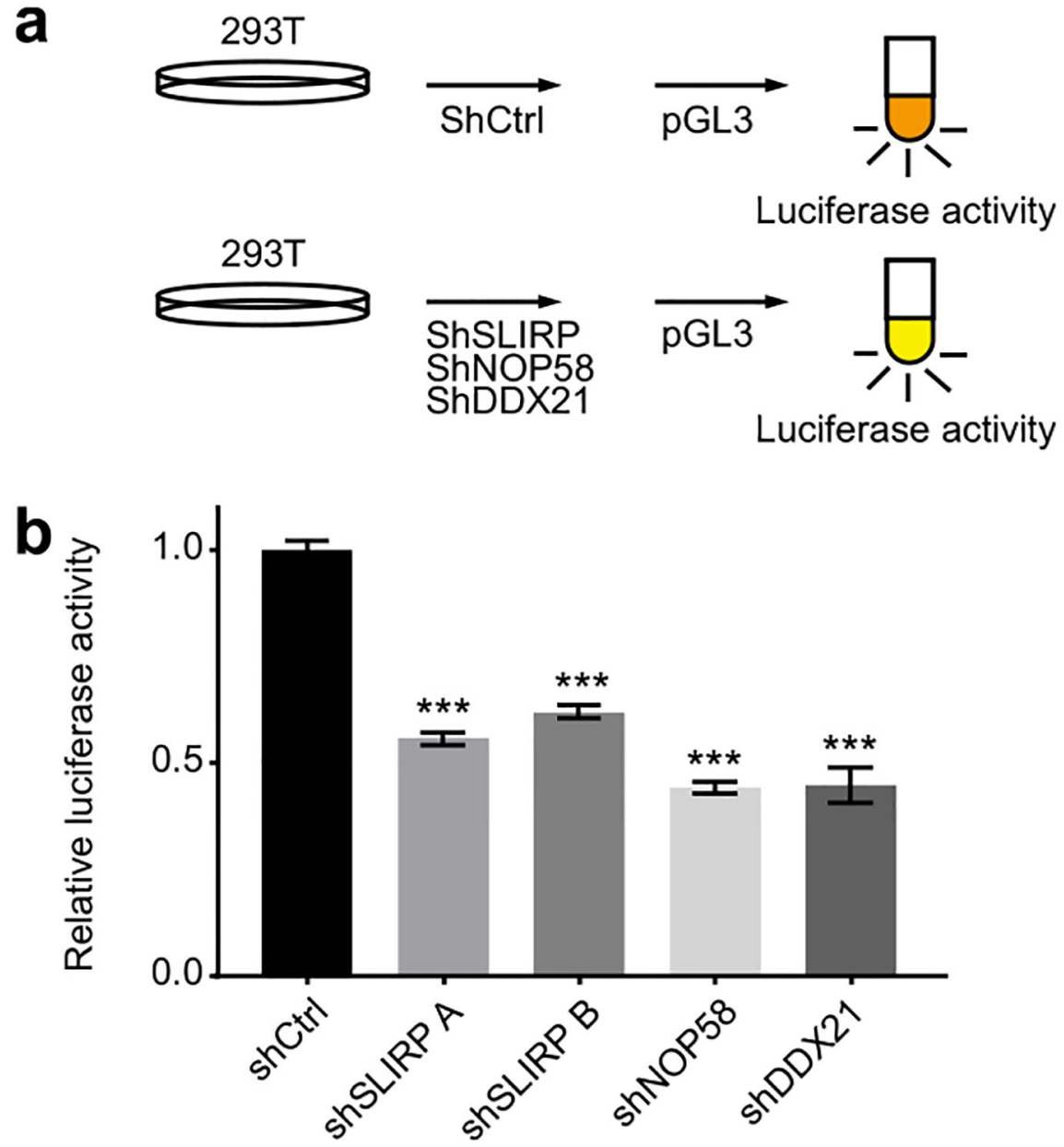

Figure 5.

SLIRP regulates translation efficiency. (a) A schematic diagram depicting the procedures for monitoring the translation efficiency using luciferase activity assay. (b) Relative luciferase activity in cells after shRNA-mediated knockdown of SLIRP, NOP58 or DDX21. 